\title{
CƠ CẤU TRỢ LựC TRONG Hệ THỐNG THOÁT HIỂM CCD
}

\author{
PHAN CHÍ CHÍNH \\ Trưòng Đại học Công nghiệp thành phố Hồ Chí Minh; \\ phanchichinh@iuh.edu.vn
}

Tóm tắt. Bài báo này nghiên cứu và giải quyết vấn đề trợ lực trong hệ thống thoát hiểm Cabin - Cáp Đu trượt (hệ thống $\mathrm{CCD}$ ) cho các tòa nhà cao tầng. Tiếp cận để giải quyết vấn đề này của chúng tôi là dùng nguyên tắc đối trọng. Dựa trên nguyên tắc đối trọng, chúng tôi xây dựng kết cấu bộ trợ lực thủy lực (BTLTL) cho hệ thống CCD. Bộ trợ lực thủy lực này được cấu trúc dựa trên ứng dụng tích hợp nguyên lý đối trọng với hiệu ứng thủy lực qua van tiết lưu.

Từ khóa. CCD, hệ thống CCD, nguyên lý đối trọng, BTLTL, bộ trợ lực thủy lực

\section{WEIGHT- ASSIST STRUCTURE IN ESCAPE SYSTEM CCD}

\begin{abstract}
This study researches and resolves weight-assist issue of Cabin - Cable - Sliding system (CCD system) in high buildings. Our approach is applying the counterpoise principle. Basing on the counterpoise principle, we built Hydraulic Weight- Assist structure (BTLTL) for CCD system. This BTLTL is structured basing on intergrated applying the counterpoise principle with hydraulic throttle effect.
\end{abstract}

Keywords. CCD system, counterpoise principle, BTLTL, Hydraulic Weight- Assist structure

\section{DÃ̃N NHậP VÀ ĐẶT VẤN ĐỀ}

Hiện nay, đã có nhiều giải pháp để cứu hộ cho nhà cao tầng, một trong các phương pháp đó có thể kể ra như ống tuột, đệm hơi, dây thang, xe thang. Tuy nhiên, các giải pháp này vẫn còn một số nhược điểm như đệm hơi, yêu cầu phải nhảy từ trên cao xuống gây tâm lý sợ hãi, dây thang và xe thang khó sử dụng trong trường hợp người bị nạn đã không còn bình tĩnh hoặc người tàn tật, già yếu khó di chuyển.

Chúng tôi đề xuất giải pháp chế tạo một hệ thống thoát hiểm Cabin- Cáp - Đu trượt $(\mathrm{CCD})$ cho các tòa nhà cao tầng. Nguyên lý thoát hiểm liên quan đển cấu tạo của hệ thống thoát hiểm CCD. Thiết bị chính của hệ thống thoát hiểm CCD bao gồm nhiều cặp Cáp - Cabin và các Đu trượt. Các cabin được phân bố trong các tầng của tòa nhà với mật độ phù hợp. Nguyên tắc hoạt động chung của hệ thống CCD là khi hỏa hoạn xảy ra, người thoát hiểm sẽ đứng trên đu trượt trong cabin, điều khiển tốc độ và trượt cùng đu trượt xuống đất.

Bài báo này tập trung trình bày một trong các bộ phận cốt lõi được sử dụng trong hệ thống $\mathrm{CCD}$, đó là cơ cấu trợ lực. Cơ cấu trợ lực trong hệ thống CCD được chúng tôi xây dựng dựa trên nguyên tắc đối trọng vẫn thường hay dùng trong máy nâng chuyển. Dựa trên nguyên tắc đối trọng, chúng tôi xây dựng kết cấu bộ trợ lực thủy lực (BTLTL) cho hệ thống CCD. Bộ trợ lực thủy lực này được cấu trúc dựa trên ứng dụng tích hợp nguyên lý đối trọng với hiệu ứng thủy lực qua van tiết lưu.

Bằng cách ứng dụng cơ cấu trợ lực được thiết kế phù hợp với trọng lượng của tải trọng (1 người và đu trượt) trong vận hành, việc điều khiển đu trượt hạ xuống được an toàn và tạo cảm giác an tâm cho người thoát hiểm khi sử dụng hệ thống CCD.

\section{NỘI DUNG KHOA HỌC}

\section{Nguyên tắc đối trọng kết nối trong hệ thông CCD}

Để điều khiển tốc độ khi trượt hạ xuống và thử nghiệm các hiệu ứng trợ lực trong giải pháp thoát hiểm, hệ thống CCD được tích hợp 'cơ cấu trợ lực' (gọi tắt là Bộ Trợ Lực - BTL).Ý tưởng của đề xuất BTL mô tả như Hình 1. 


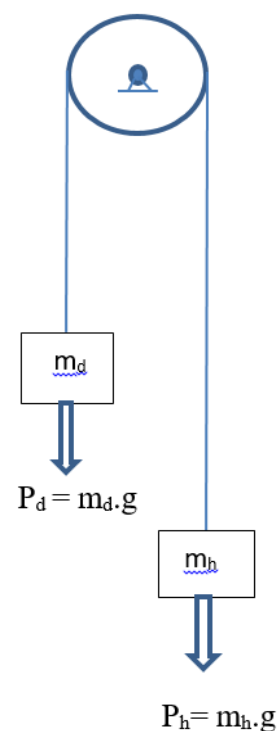

Hình 1. Sơ đồ nguyên lý đối trọng ứng dụng trong BTL

Trong hình 1: $\mathrm{m}_{\mathrm{d}}$ là khối lượng của đối trọng $(\mathrm{kg}) ; \mathrm{P}_{\mathrm{d}}$ là trọng lượng của đối trọng $(\mathrm{N}) ; \mathrm{P}_{\mathrm{h}}$ là trọng lượng hạ xuống $(\mathrm{N}) ; \mathrm{m}_{\mathrm{h}}$ là khối lượng hạ xuống $(\mathrm{kg}) ; \mathrm{g}=9,81 \mathrm{~m} / \mathrm{s}^{2}$ là gia tốc trọng trường.

Trong trường hợp này, vận tốc chạm đất của khối lượng $\mathrm{m}_{\mathrm{h}}$ có thể tính toán dựa trên phương trình định luật 2 của Newton. Nếu gọi $h$ là chiều cao của tầng nhà, vận tốc sẽ là:

$$
v=\sqrt{2 a h}
$$

Trong đó $a$ là gia tốc rơi của toàn bộ hệ. Với $a=\frac{\left(m_{h}-m_{d}\right)}{m_{h}+m_{d}} g$. Tùy thuộc vào vận tốc mong muốn, khối lượng $\mathrm{m}_{\mathrm{d}}$ có thể tính toán được. Nhiệm vụ là chọn khối lượng phù hợp để vận tốc chạm đất không gây nguy hiểm cho người.

Khi đề cập ứng dụng nguyên lý đối trọng trong hệ thống $\mathrm{CCD}$, để phân tích hiệu quả ứng dụng chúng tôi xây dựng sơ đồ kết nối đối trọng với đu trượt và khảo sát hiệu ứng bằng các tính toán về trọng lực (trọng lượng các thành phần).

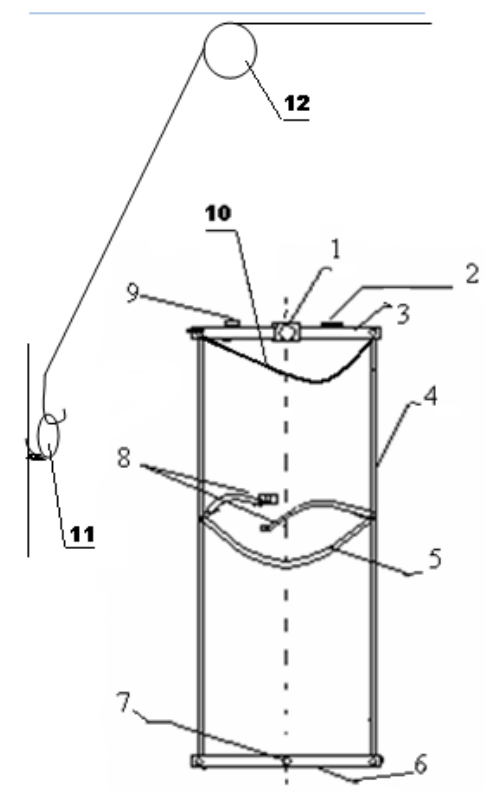

Hình 2. Đu trượt được kết nối đối trọng 
1. cơ cấu kẹp chặt và điều khiển tốc độ; 2 . nút điều khiển tốc độ; 3 . thanh trên; 4. chạc dù liên kết 2 thanh; 5 . dây an toàn; 6 . thanh dưới; 7 . định hướng dây cáp chính; 8 . khóa an toàn; 9 . nút ấn nhả cáp; 10. chạc dù móc đối trọng; 11 . móc giữ cáp đối trọng; 12. ròng rọc

Việc kết hợp đu trượt với đối trọng được mô tả theo hình 2 . Trước khi người thoát hiểm đứng vào đu trượt thì đu trượt đã được gắn chặt vào sợi cáp chính làm đường trượt (đường cáp chính làm đường trượt biểu diễn bằng nét đứt trên hình 2 ), người sử dụng dùng tay móc dây cáp đối trọng vào sợi dây chạc dù số 10 gắn vào 2 đầu thanh trên đu trượt. Trước đó cáp đối trọng được móc vào móc 11 cố định trên thành cabin.

Khối lượng hạ xuống bao gồm khối lương của người $\left(\mathrm{m}_{\mathrm{ng}}\right)$ và khối lượng của bản thân đu trượt $\left(\mathrm{m}_{\mathrm{DT}}\right)$.Trọng lượng hạ xuống bằng tổng trọng lượng của người thoát hiểm và tự trọng của đu trượt:

Ở đây

$$
P_{h}=P_{n g}+P_{D T}
$$

$P_{D T}-$ là tự trọng của đu trượt với $\mathrm{P}_{\mathrm{DT}}=\mathrm{m}_{\mathrm{DT}} \mathrm{g}$

$P_{n g}-$ là trọng lượng của người đứng trên đu trượt với $\mathrm{P}_{\mathrm{ng}}=\mathrm{m}_{\mathrm{ng}} \mathrm{g} \mathrm{g}$

Điều kiện hạ trượt xuống của đu trượt là $P_{d}<P_{h}$ hay

$$
P_{d}<P_{n g}+P_{D T}
$$

Trọng lượng hạ xuống được điều khiển qua hệ thống $\mathrm{CCD}$ chỉ còn là hiệu số $P_{h}$ và $P_{d}$. Gọi lượng đó là $P_{h t}$

Hay

$$
P_{h t}=P_{h}-P_{d}
$$

$$
P_{h t}=\left(P_{n g}+P_{D T}\right)-P_{d}
$$

Chú ý rằng điểm bổ xung từ hình 2 so với nguyên lý ban đầu về đối trọng ở hình 1 là đu trượt được kẹp chặt vào cáp chính. Điều này cho thấy rằng đối trọng mặc dù giảm trọng lượng hạ xuống đáng kể nhưng điều khiển tốc độ (dừng hoặc cho chạy nhanh hay chậm) lại được thực hiện chính là ở cơ cấu kẹp và nhả cáp có cấu trúc gắn liền với thanh trên của đu trượt. Đối trọng hỗ trợ giảm trọng lượng hạ xuống làm sự điều khiển tốc độ hiệu quả hơn.

Khi người thoát hiểm ấn cơ cấu nhả cáp để cho đu trượt hạ xuống, tác dụng của đối trọng đã làm giảm nhẹ các lực cần điều khiển trong cơ cấu thanh trên 3 của đu trượt như hình 2 . Có thể lấy trị số ví dụ sát với thực tiễn $P_{d}=60 \mathrm{kG}(\sim 600 \mathrm{~N})$; trọng lượng $\mathrm{P}_{\mathrm{h}}=80 \mathrm{kG}$ thì $\mathrm{P}_{\mathrm{ht}}=20 \mathrm{kG}$ (tự trọng của đu trượt $\mathrm{P}_{\mathrm{DT}}=6$ $\mathrm{kG}$ ). Với trị số $20 \mathrm{kG}$, vận tốc ở chiều cao $3 \mathrm{~m}$ khi tiếp đất sẽ là $5,4 \mathrm{~m} / \mathrm{s}$. Vận tốc này tính đến với riêng tác dụng của đối trọng. Lưu ý lại một lần nữa là trong cơ cấu như hình 2 tác dụng của cơ cấu điều khiển tốc độ có thể dừng và làm chậm lại (giảm) tốc độ của đu trượt. Vận tốc giới hạn do tác dụng của đối trọng dự phòng trường hợp mất kiểm soát do mất năng lực của người thoát hiểm (hoảng loạn hoặc già, ốm vv....).

Khi đu trượt mang theo người hạ xuống, đối trọng được kéo lên và thế năng tăng lên theo độ cao. Người thoát hiểm hạ xuống đến mặt đất và dời khỏi đu trượt; khi đó đối trọng lại hồi lại vị trí và đi xuống kéo theo đu trượt trở lại cabin (thu hồi đu trượt). Điều kiện thu hồi đu trượt là trị số $\mathrm{P}_{\mathrm{d}}$ lớn hơn $\mathrm{P}_{\mathrm{DT}}$.

\section{Kết cấu thủy lực để thay thế đối trọng}

Để giảm kích thước của hệ thống do đối trọng có khối lượng lớn dần theo độ cao, chúng tôi xây dựng kết cấu sự dụng hiệu ứng của van tiết lưu như Hình 3 .

Trong trường hợp này, chúng ta dùng tiết lưu để giảm tốc độ di chuyển của hệ thống, và lò xo dùng để phục hồi hệ thống thủy lực và đu trượt quay trở về vị trí ban đầu (đu trượt được kéo trở lại cabin). Như vậy độ dài xy lanh là:

$$
l=\frac{h}{i}
$$

Trong đó $i$ là tỉ số truyền của hệ. Trong trường hợp nhà có độ cao 10 mét, xy lanh dài $400 \mathrm{~mm}$ thì tỉ số truyền cần thiết là 25 . Độ dài xi lanh trong tính toán ở trên tương ứng đoạn di chuyển cơ bản của Piston là Hcb trong Hình 3. 


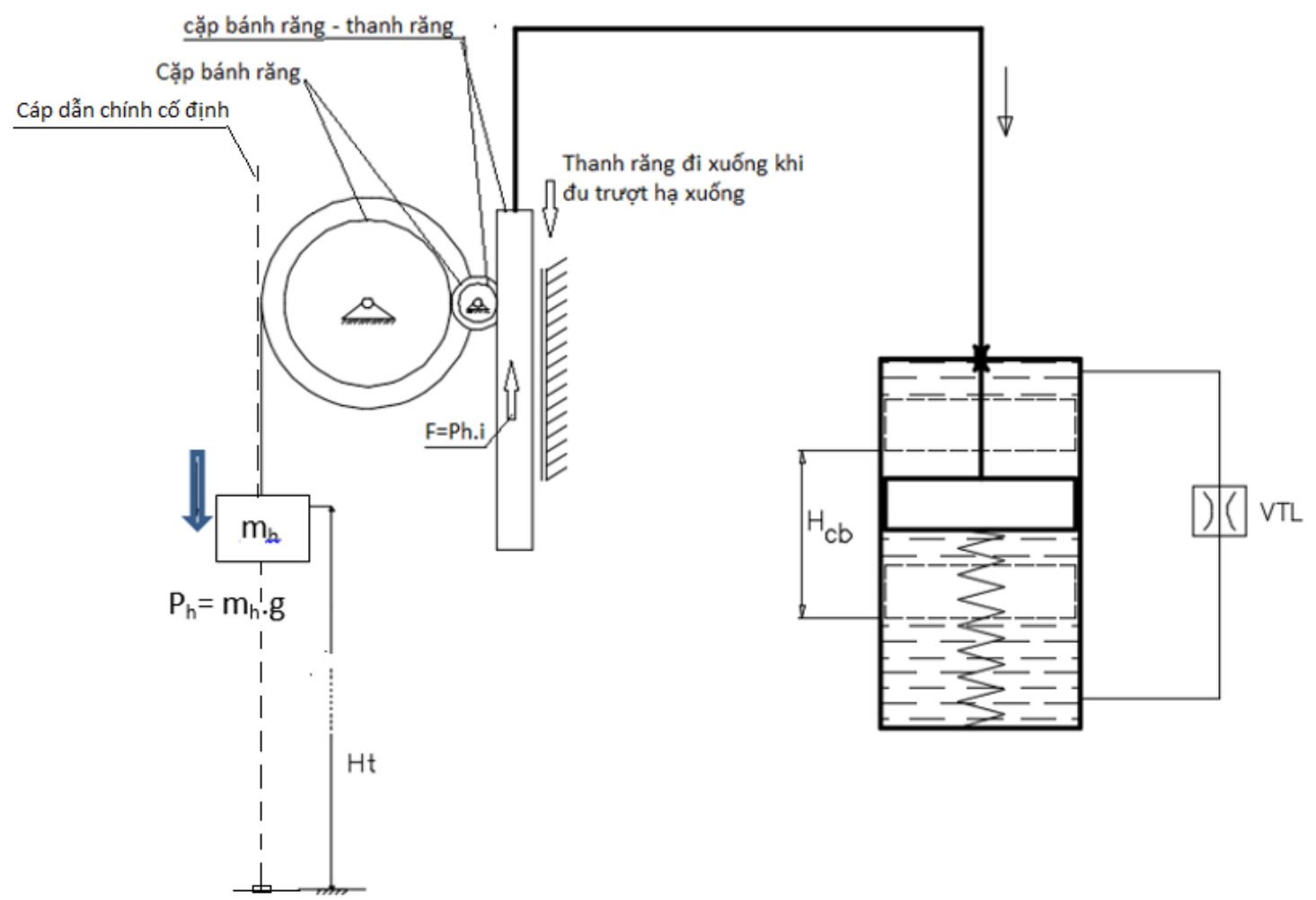

Hình 3. Kết cấu thủy lực thay thế đối trọng

\section{KẾT LUẬN}

Kết quả của nghiên cứu được đề cập có thể đúc kết như sau:

- Hệ thống CCD sử dụng nguyên lý đối trọng được tính toán khối lượng phù hợp để vận tốc chạm đất không gây nguy hiểm cho người.

- Kết cấu thủy lực sử dụng van tiết lưu để thay thế khối lượng của đối trọng; dùng tiết lưu để giảm tốc độ di chuyển của hệ thống. Nhiệm vụ xây dựng kết cấu là tính độ dài xi lang và tỷ số truyền của hệ thống.

Sử dụng nguyên lý đối trọng làm giảm trọng lượng hạ xuống giúp điều khiển tốc độ của đu trượt dễ dàng thực hiện hơn. Hay có thể hiểu là người thoát hiểm nhờ đó chủ động giảm tốc độ hoặc dừng lại khi tác động vào cơ cấu điều khiển tốc độ ở thanh trên đu trượt. Kết quả tính toán khối lượng đối trọng tương ứng tốc độ tiếp đất an toàn và sử dụng hiệu ứng tiết lưu làm giảm tốc độ di chuyển của hệ thống là những kết quả cụ thể của nghiên cứu trong bài báo này.

\section{TÀI LIỆU THAM KHẢO}

[1] Nguyễn Thượng Hiền, Giáo Trình Kỹ Thuật Thủy Khí. NXB Khoa Học Và Kỹ Thuật Hà Nội- 2011.

[2] Nguyễn Hữu Lộc, ‘Cơ Sở Thiết Kế Máy’. NXB Đại Học Quốc Gia TP.HCM 2007.

[3] Trương Quốc Thành - Phạm Quang Dũng, ‘Máy Và Thiết Bị Nâng Chuyển’. NXB Khoa Học Và Kỹ Thuật Hà Nội 2003. 